\title{
Estrategias lectoras y desempeño en pruebas de estudiantes universitarios en textos narrativos
}

Reading strategies and test performance of undergraduate students in narrative texts

Recibido: 10 de setiembre 2020, evaluado: 15 de enero 2021, aceptado: 5 de abril 2021

\author{
Alejandra Platas-García \\ https://orcid.org/0000-0002-5150-0668 \\ aplatasg@gmail.com
}

Centro de Lenguas Extranjeras, Facultad de Lenguas,

Benemérita Universidad Autónoma de Puebla, México

\section{J.-Martín Castro-Manzano}

https://orcid.org/0000-0003-2227-921X

josemartin.castro@upaep.mx

Facultad de Filosofía, UPAEP Universidad, Puebla. México.

\section{Verónica Reyes-Meza}

https://orcid.org/0000-0002-2745-4032

veronica.reyesm@uatx.mx

Centro Tlaxcala de Biología de la Conducta,

Universidad Autónoma de Tlaxcala. México. 


\section{Resumen}

Esta investigación tuvo como objetivos comparar tanto las estrategias de lectura como el desempeño en una prueba de comprensión lectora inferencial de estudiantes universitarios, mujeres y hombres, de tres áreas académicas usando textos narrativos. Se realizaron dos experimentos que corresponden a investigaciones cuantitativas, estudios descriptivos de corte transversal, clasificando a los estudiantes por su área académica (en un experimento) y por su género (en el otro). Los participantes del primer experimento fueron 33 universitarios pertenecientes a las siguientes áreas académicas: Cs. Económico-Administrativas (11 estudiantes); Cs. Exactas e Ingenierías (11); Cs. Sociales y Humanidades (11); mientras que la muestra del segundo experimento se conformó por 18 estudiantes (9 mujeres y 9 hombres). Los instrumentos empleados fueron el Cuestionario de Estrategias Metacognitivas de Comprensión de Textos Narrativos y una prueba de comprensión lectora inferencial diseñada para esta investigación. Se encontró, con respecto a las estrategias de lectura, que los estudiantes pertenecientes al área de Cs. Exactas e Ingenierías emplean menos las estrategias de creatividad en comparación con los estudiantes de las áreas de Cs. EconómicoAdministrativas y Cs. Sociales y Humanidades y esta diferencia fue estadísticamente significativa empleando una prueba de ANOVA $(f=8.811, n=33, p=.001)$. Sin embargo, no se encontraron diferencias estadísticamente significativas en relación al desempeño en la prueba. En trabajos futuros se podrían ampliar las muestras para comparar estos resultados.

Palabras clave: prueba de lectura; respuestas abiertas; opción múltiple; área académica; género

\section{Summary}

In this research we tried to compare both reading strategies and performance in an inferential reading comprehension test within undergraduate students, women and men, from three academic areas using narrative texts. Two experiments were carried out corresponding to quantitative investigations, descriptive cross-sectional studies, classifying the students by their academic area (in one experiment) and by their gender (in the other). The participants of the first experiment were 33 undergraduate students belonging to the following academic areas: Economic-Administrative Sciences (11 students); Exact Sciences and Engineering (11); Social Sciences and Humanities (11); while the sample of the second experiment was made up of 18 
students (9 women and 9 men). Two instruments were employed: the Questionnaire of Metacognitive Strategies to Comprehend Narrative Texts, and an inferential reading comprehension test specially designed for this research. We found, with respect to reading strategies, that students belonging to the area of Exact Sciences and Engineering employ less creativity strategies when compared to students in the Economic-Administrative, Social Sciences and Humanities. This difference being statistically significant using an ANOVA test $(f=8.811, n=33, p=.001)$. However, no statistically significant differences were found in relation to test performance. In future work, the samples could be expanded to further compare these results.

Keywords: reading tests; open-ended responses; multiple-choice; academic area, gender 


\section{Introducción}

Dado que la comprensión lectora no se puede medir de manera fidedigna mediante observaciones directas, se tiene que evaluar a través de tareas que ofrezcan evidencia de cierto grado de comprensión (Pérez Zorrilla, 2005; cf. Pearson y Cervetti, 2017). Ahora, esta evaluación indirecta de la comprensión lectora a través de tareas o pruebas escritas suele suponer distintos tipos de preguntas, a saber: preguntas abiertas, en las que no se presentan las respuestas a los estudiantes y estos contestan según su criterio; y preguntas cerradas, las cuales contienen las respuestas y los estudiantes seleccionan entre distintas opciones de respuesta (Torres, Paz y Salazar, 2006).

Por otra parte, el nivel de comprensión lectora solicitado a los estudiantes suele estar indicado por la forma de la(s) pregunta(s) presentada(s) después de un texto específico. Para el interés de este trabajo, elaboramos preguntas que indican la realización de inferencias (deductivas, inductivas o abductivas). Además, seleccionamos fragmentos de textos narrativos porque los estudiantes emplearían poco tiempo en su lectura y porque podrían elaborar inferencias como leemos en la cita a continuación: "los textos narrativos generan un mayor número de inferencias requiriendo un tiempo de lectura menor, mientras que los textos expositivos requieren de un mayor tiempo de procesamiento debido al mayor esfuerzo de integración que requiere la información leída" (Escudero y León, 2007, p. 322).

Asimismo, los esquemas que poseen los lectores por sus conocimientos previos (debido a su cultura en relación al contenido del texto) ejercen una profunda influencia en la forma como el discurso será comprendido, aprendido y recordado (Steffensen, Joag-Dev y Anderson, 1979). Se podría suponer, entonces, de forma análoga, que los conocimientos previos de los estudiantes (saberes o estrategias especializadas) no en cuanto a su herencia cultural, sino a los conocimientos adquiridos debido al área académica a la que pertenecen (cf. Alderson y Urquhart, 1988), posibilitarían usos diferenciados de las estrategias de lectura que estos emplean, así como desempeño en pruebas de comprensión lectora también diferenciado empleando textos narrativos. Unido a esto, se sabe que los hábitos de lectura de los estudiantes dependen de su perfil académico (Gilardoni, 2006).

Ahora, estos usos de las estrategias lectoras y desempeño diferenciados al leer textos narrativos podrían observarse también entre mujeres y hombres porque se sabe que las mujeres prefieren leer esta tipología de textos: en un estudio sobre la lectura digital, West y Chew (2015) reportan que las mujeres prefieren leer ficción, por ejemplo, novelas románticas; 
mientras que los hombres se interesan más por la no ficción y leen libros, por ejemplo, de política.

Dado los supuestos anteriores, en este artículo reportamos los resultados de dos experimentos con el objetivo de comparar tanto las estrategias de lectura, como el desempeño en una prueba de comprensión lectora inferencial empleando textos narrativos en grupos de estudiantes universitarios clasificados conforme a (1) su área académica y (2) su género.

Se presentan a continuación los ejes teóricos que sustentan esta investigación y los cuales se enfocan en los siguientes temas: los textos narrativos y las estrategias metacognitivas que se emplean para leerlos; la formulación de preguntas inferenciales; las respuestas cerradas y abiertas en pruebas de comprensión lectora.

\section{Textos narrativos y estrategias lectoras}

Los textos narrativos suelen incluir los siguientes elementos: la caracterización de un personaje, la perspectiva de un protagonista, la implicación de secuencias de hechos que se presentan comúnmente como una cadena causal y con un orden temporal, el marco organizativo de la historia que le permite al lector explicar las situaciones en las que se ven envueltos los personajes, así como las posibles consecuencias que pueden derivarse (Escudero y León, 2007). Ejemplos de textos narrativos son las narraciones de hechos históricos, las autobiografías, las historias de ficción, los cuentos, las fábulas, las leyendas.

La lectura de textos narrativos requiere de "la reflexión, el análisis, la empatía y la apropiación personal del texto" (Larrañaga y Yubero, 2015, p. 21). Así, cuando los estudiantes leen textos narrativos aplican distintas estrategias metacognitivas que, siguiendo a Larrañaga y Yubero (2015), se clasifican en:

- Estrategias Globales de Lectura que permiten al lector monitorear y controlar el proceso de comprensión del texto. Por ejemplo: detenerse en algunos párrafos del libro para reflexionar; volver a retomar el hilo cuando se pierde la concentración.

- Estrategias de Personalización que reflejan la apropiación personal del texto y la aplicación del discurso a la propia vida. Por ejemplo, analizar los personajes e imaginar cómo se sienten; relacionar y comparar el texto con la vida real.

- Estrategias de Creatividad que hacen posible para el lector la realización de procesos inferenciales de reconstrucción del texto. Por ejemplo, plantearse por qué 
los personajes se comportan de un determinado modo; adivinar qué va a pasar conforme se va leyendo.

\section{Formulación de preguntas inferenciales}

La formulación de preguntas inferenciales es un elemento importante al momento de diseñar una prueba de comprensión lectora. De hecho, "toda la actividad mental que se genera cuando tratamos de comprender un discurso, sea cual sea su naturaleza, requiere de la activación de inferencias" (Escudero y León, 2007, p. 314). Ahora, la formulación misma de las preguntas inferenciales es un indicador de las inferencias realizadas previamente por los diseñadores de las pruebas, es decir, de sus creencias de que algo se concluya de forma necesaria, probable o plausible. Una referencia que puede iluminar este punto la leemos a continuación:

Hay unos presupuestos que hacemos al formular una pregunta. Si alguien pregunta seriamente “¿Quién mató a César?” presupone que alguien lo mató (un presupuesto positivo) y que alguien no lo mató (presupuesto negativo). Una pregunta muestra creencias de quién pregunta precisamente a través de los presupuestos positivos y negativos; en este sentido las oraciones interrogativas pueden ser usadas para comunicar información. (Campos, 2016, p. 125).

El esfuerzo que deben realizar los estudiantes al resolver una prueba de comprensión lectora con preguntas inferenciales es mayor que el esfuerzo que realizan si responden preguntas para las cuales sería suficiente copiar la información que está explícita en el texto (como el lugar donde se desarrollan las acciones o el tiempo en que trascurren las mismas), es decir, una lectura literal (cf. Méndez, 2006). En cambio, gracias a una lectura inferencial "podemos desvelar lo 'oculto' de un mensaje, leer entre líneas, hacer explícita en nuestra mente la información implícita del pasaje” (Escudero y León, 2007, p. 314).

La clasificación de inferencias que hemos empleado en este trabajo es tomada de la lógica. Existen tres tipos de inferencias, a saber, deducción (conclusión que se sigue necesariamente de un conjunto de datos), inducción (conclusión que se sigue probablemente de un conjunto de hechos) y abducción (conclusión que se sigue como la explicación más plausible). Para una explicación detallada de estas inferencias véase Adler y Rips (2008). 


\section{Respuestas cerradas y abiertas en pruebas de comprensión lectora}

Las pruebas de respuestas cerradas (por ejemplo, opción múltiple, verdadero o falso) son ampliamente utilizadas debido a la facilidad con que se califican y se aplican. Específicamente, en los reactivos de opción múltiple, se proporcionan tres tipos de información a los estudiantes: un radical, que plantea una pregunta; la respuesta correcta, que responde a la pregunta de manera acertada; y distractores, tres o cuatro afirmaciones que podrían ser la respuesta correcta, pero que no lo son (Arends, 2007).

Es muy importante dar retroalimentación de las respuestas correctas a los estudiantes después de una prueba de opción múltiple porque así se reduce el efecto negativo que estas pueden tener, a saber, la posibilidad de aprender información falsa a través de las afirmaciones de los distractores (Butler y Roediger, 2008).

Una crítica que se suele hacer a las pruebas de opción múltiple es que promueven el uso de la adivinación. Al respecto, Brown, Bull y Pendlebury (1997) afirman que los efectos de la adivinación podrían ser eliminados, pero si esta es una adivinación inteligente podría ser alentada, al considerarla como una habilidad útil, pues sería una especie de predicción a partir de los conocimientos previos, una suerte de abducción.

Ahora, con la tipología de pruebas de respuesta abierta es más fácil indagar sobre habilidades inferenciales complejas que con las preguntas cerradas (Intraversato y Lucisano, 2013).

Sin embargo, la corrección de las respuestas abiertas (a diferencia de las respuestas cerradas) requiere de mucho más trabajo por parte de los evaluadores en términos de la codificación de las respuestas escritas por los estudiantes, la identificación de las categorías y la asignación de corrección o incorrección de las mismas; mas, a pesar de este inconveniente, estas respuestas permiten valorar la riqueza de las ideas presentadas, las soluciones alternativas y los razonamientos seguidos por los estudiantes (Intraversato y Lucisano, 2013).

Ahora bien, una limitación importante de esta tipología de pruebas es que requieren que los estudiantes realicen una actividad de producción escrita que puede ser considerada una dificultad que se añade al hecho de dar una respuesta, e incluso puede ser considerada una desventaja para quienes no han desarrollado suficientemente la habilidad de producción escrita (de hecho, al evaluar estas respuestas se ignoran errores de ortografía y gramática) (Intraversato y Lucisano, 2013). 


\section{Método}

Realizamos dos experimentos con el objetivo de comparar tanto las estrategias de lectura, como el desempeño en una prueba de comprensión lectora inferencial empleando textos narrativos en grupos de estudiantes universitarios clasificados conforme a (1) su área académica y (2) su género. Estos experimentos se diferencian en la muestra seleccionada y la tipología de respuesta en la prueba de comprensión lectora. Ambos experimentos corresponden a investigaciones cuantitativas, descriptivas, de corte transversal.

Para lograr el objetivo en cada experimento procedimos en dos fases: en una primera fase, identificamos el comportamiento lector y las estrategias de lectura de textos narrativos de los estudiantes clasificados conforme a su área académica y su género; y como segunda fase, reportamos los resultados de una prueba de comprensión lectora inferencial empleando textos narrativos. Para la clasificación por área académica empleamos la respuesta abierta, mientras que, para la clasificación por género, empleamos la respuesta cerrada de opción múltiple.

\section{Muestra}

\section{Clasificación por área académica}

Los participantes fueron 33 estudiantes universitarios (22 mujeres y 11 hombres) con una edad promedio de 21.2 años ( $\mathrm{SD} \pm 1.5$ ), todos inscritos en distintos semestres y programas académicos de licenciatura que, de acuerdo con la clasificación de la universidad de origen, se agrupan en las siguientes áreas académicas: Cs. Económico-Administrativas (11 estudiantes), Cs. Exactas e Ingenierías (11), Cs. Sociales y Humanidades (11).

\section{Clasificación por género}

Los participantes fueron 18 estudiantes universitarios ( 9 mujeres y 9 hombres) con una edad promedio de 21.3 años ( $\mathrm{SD} \pm 1.9$ ), todos inscritos en distintos semestres y programas académicos de licenciatura que se agrupan en las siguientes áreas académicas: Cs. EconómicoAdministrativas (4 estudiantes), Cs. Exactas e Ingenierías (11), Cs. Sociales y Humanidades (2), Cs. Naturales y de la Salud (1). 


\section{Instrumentos}

\section{Primero}

Para evaluar el comportamiento lector empleamos la técnica que proponen Elche, SánchezGarcía y Yubero (2019), quienes presentan las siguientes preguntas a los lectores: (1) "Aparte de tus lecturas para estudiar, ¿con qué frecuencia dedicas tiempo a la lectura voluntaria?” Posibles respuestas: nunca; casi nunca; al menos una vez al semestre; al menos una vez al mes; al menos una vez a la semana; todos o casi todos los días. (2) “Cuál es el número de libros que has leído por placer durante el último año?” Posibles respuestas: ninguno; 1 o 2; de 3 a 5 ; de 6 a 10; de 11 a 15; de 16 a 20; más de 21. Posteriormente, los autores cruzan las respuestas de estas preguntas para clasificar a los lectores como:

1. No lectores: no leen nunca o casi nunca; y leen entre 0 y 2 libros al año.

2. Lectores ocasionales: leen alguna vez al semestre o al mes; y leen entre 3 y 10 libros al año.

3. Lectores frecuentes: leen casi todos los días o más de una vez a la semana; y leen más de 10 libros al año.

4. Falsos lectores: aquellos que en una respuesta se muestran como lectores y en la otra como no lectores (es decir, quien no es lector, pero quiere aparentar serlo).

\section{Segundo}

Para evaluar la frecuencia de uso de las estrategias metacognitivas de comprensión de textos narrativos empleamos el Cuestionario de Estrategias Metacognitivas de Comprensión de Textos Narrativos (CEMCo-TeN) de Larrañaga y Yubero (2015). Este cuestionario fue empleado en estudiantes universitarios, cuenta con 17 enunciados con una escala de Likert que va de 1 (nunca), 2 (casi nunca), 3 (casi siempre) a 4 (siempre). Clasifica los ítems en tres tipos de estrategias, a saber: Estrategias Globales de Lectura (por ejemplo: "Cuando no entiendo el texto lo vuelvo a leer para aumentar mi comprensión"), Estrategias de Personalización (por ejemplo: "Analizo los personajes y me imagino cómo se sienten"), y Estrategias de Creatividad (por ejemplo: "Me voy imaginando cómo me gustaría que avanzara el relato").

\section{Tercero}

Para conocer el desempeño en una tarea de comprensión lectora empleando textos narrativos diseñamos para esta investigación una prueba de comprensión lectora inferencial con textos 
narrativos. Las características son las siguientes: la prueba tiene 12 reactivos que se componen (cada uno) de un texto narrativo que va de longitudes de 34 palabras como mínimo a 144 palabras como máximo; se sigue de una pregunta que requiere un tipo de inferencia deductiva (4 reactivos), inductiva (4 reactivos) o abductiva (4 reactivos). Las temáticas de los textos son diversas.

Para la clasificación de la muestra por área académica, el tipo de respuesta solicitada fue respuesta abierta; mientras que para la clasificación por género fue respuesta cerrada (opción múltiple). Presentamos a continuación el ejemplo de un reactivo en cada tipología de respuesta (a los estudiantes no les dimos la referencia con los datos del autor del fragmento):

\section{Fragmento:}

"Nunca podremos olvidar aquella espera dramática en el anochecer del día 1 de diciembre de 1963, ocultos en el fondo de una rambla perdida en el hosco paraje del término villenense y a la luz de unas hogueras que hacía brillar, con destellos intermitentes, el oro de unos objetos que habían permanecido ocultos a las miradas humanas durante miles de años” (Soler García, 2005).

\section{Pregunta:}

De acuerdo al texto anterior, ¿cuál podría ser el acontecimiento que mejor explicaría el fragmento?

- Respuesta abierta.

- Respuesta cerrada:
(A) El robo histórico de objetos de oro de un importante museo.
(B) La espera dramática del inicio de una rebelión el día 2 de diciembre.
(C) La observación de un ritual ancestral por parte de testigos ocultos.
(D) El descubrimiento de un tesoro formado por varias piezas de oro. (Correcta).

\section{Procedimiento}

Los participantes firmaron un documento en el que consintieron que se usaran sus respuestas para la investigación; asimismo, se les informó de la confidencialidad con la que se conservarían sus datos personales. La aplicación de los tres instrumentos fue de forma presencial utilizando lápiz y papel, los estudiantes emplearon entre 30 y 40 minutos aproximadamente para responder los tres instrumentos. 
Técnicas de análisis de datos

El procedimiento para analizar los datos fue el siguiente. Para el comportamiento lector, realizamos análisis de frecuencias; mientras que para las estrategias de lectura de textos narrativos evaluamos la normalidad de la distribución de los datos mediante la prueba ShapiroWilk, y como los datos pasaron la prueba de normalidad, empleamos la prueba paramétrica del ANOVA que se emplea para comparar las medianas obtenidas en tres o más grupos para la clasificación por área; mientras que, para la clasificación por género, empleamos la prueba paramétrica t de Student para dos muestras independientes.

Del mismo modo, para la comparación de los resultados de los estudiantes en la prueba de comprensión lectora, para la clasificación por áreas académicas, evaluamos la normalidad de la distribución de los datos mediante la prueba Shapiro-Wilk, y como los datos pasaron la prueba de normalidad para las inferencias deductivas empleamos la prueba paramétrica del ANOVA; mientras que los datos no pasaron la prueba de normalidad para las inferencias inductivas ni abductivas. En estos últimos casos empleamos la prueba de Kruskal-Wallis porque es una alternativa no paramétrica al ANOVA para comparar las medianas obtenidas en tres o más grupos.

Por último, para la comparación de los resultados de los estudiantes en la prueba de comprensión lectora, para la clasificación por género, evaluamos la normalidad de la distribución de los datos mediante la prueba Shapiro-Wilk, pero como los datos no pasaron la prueba de normalidad en los tres tipos de inferencia, empleamos la prueba U de Mann-Whitney, la cual es una alternativa no paramétrica a la $\mathrm{t}$ de Student para comparar dos muestras independientes. Para realizar estas pruebas utilizamos GraphPad Prism, 5.01 (GraphPad Software Inc., San Diego, USA).

\section{Asignación de valor de las respuestas en las pruebas}

Para comparar el desempeño en la clasificación de la muestra por área académica establecimos los siguientes criterios de asignación de un valor numérico a las respuestas abiertas de los estudiantes en la prueba de comprensión lectora (ver tabla 1). 


\section{Tabla 1}

Criterios de asignación de valor numérico a las respuestas abiertas

\begin{tabular}{lllc}
\hline Etiqueta & Descripción & Valor \\
\hline Válida & Corresponde a la respuesta que & 1 \\
& contiene una conclusión relevante a & \\
& partir de la información presente en el & \\
& fragmento de texto. & \\
\hline Válida & Corresponde a la respuesta que & \\
incompleta & contiene una conclusión relevante a & \\
& partir de una parte de la información & \\
& presente en el fragmento de texto. Es & \\
\hline Inválida & una conclusión incompleta. & \\
& Corresponde a la respuesta que & \\
& contiene una conclusión no relevante a & \\
& partir de la información presente en el & \\
\hline
\end{tabular}

Nota: Entendemos por "conclusión relevante" aquella respuesta a una pregunta inferencial que se deriva del fragmento del texto narrativo y del contexto proporcionado en este (cf. Méndez, 1995).

Fuente: elaboración propia.

Mientras que para comparar el desempeño en la clasificación de la muestra por género, consideramos la selección de la respuesta correcta en la prueba de comprensión lectora de opción múltiple con valor de 1 y la selección de un distractor con valor de 0 .

\section{Resultados}

Presentamos los resultados a continuación conforme a las dos fases mencionadas. Para facilitar la lectura hemos abreviado las áreas académicas de la siguiente forma: Cs. EconómicoAdministrativas (CEA); Cs. Exactas e Ingenierías (CEI); y Cs. Sociales y Humanidades (CSH). 


\section{Primera fase}

Para los resultados del comportamiento lector mostramos a continuación las frecuencias obtenidas que muestran que, en la mayoría de los casos, los estudiantes son "falsos lectores" tanto en la clasificación por área académica como por género (ver figuras 1 y 2).

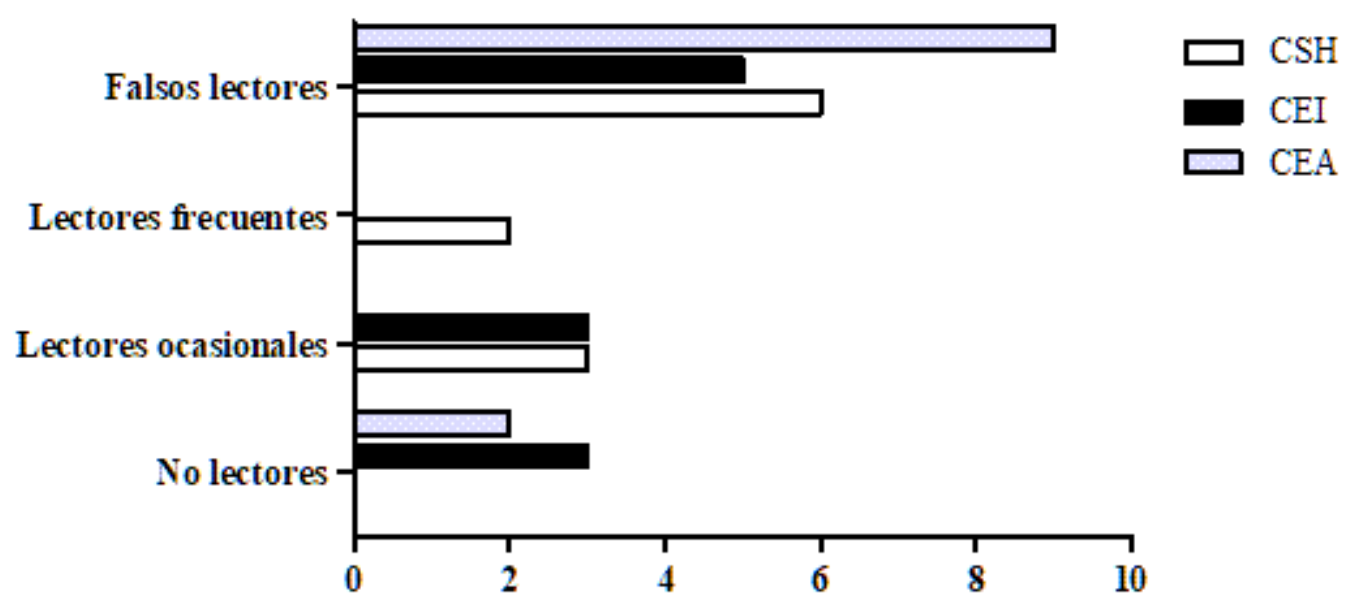

Figura 1. Comportamiento lector de los estudiantes universitarios de las tres áreas académicas.

Fuente: elaboración propia.

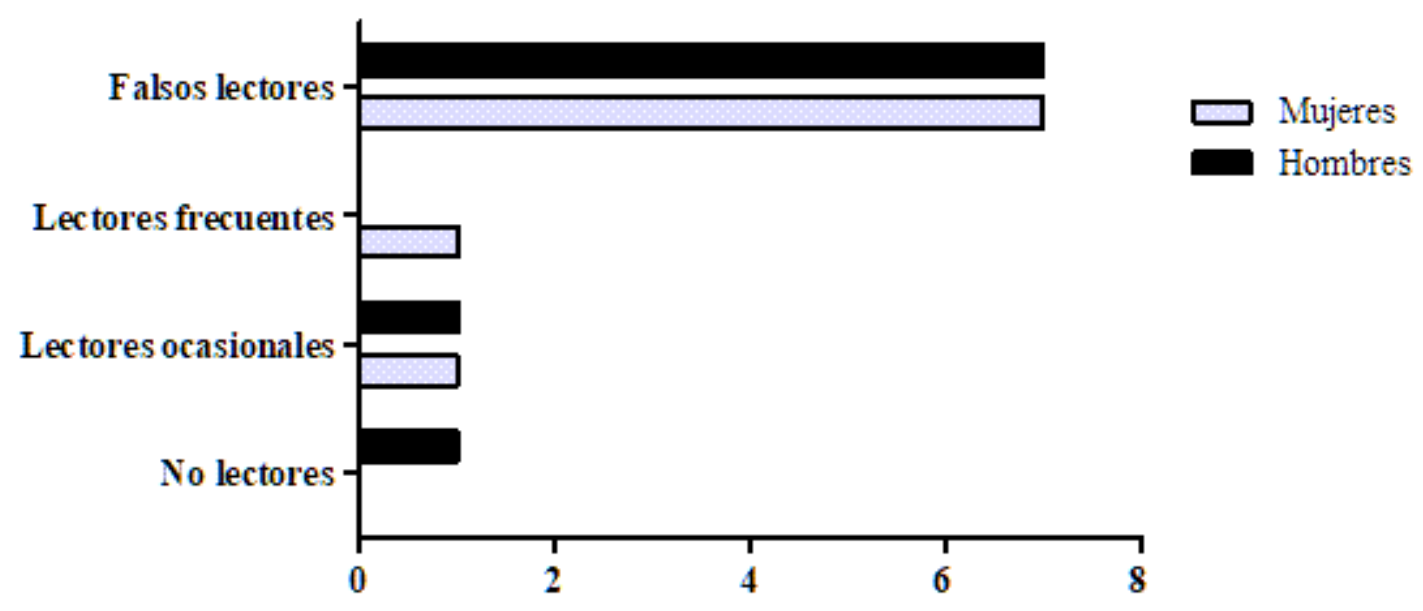

Figura 2. Comportamiento lector de los estudiantes universitarios mujeres y hombres. Fuente: elaboración propia.

Para los resultados de las estrategias lectoras de textos narrativos, comparamos la frecuencia de uso de las estrategias metacognitivas de comprensión de textos narrativos entre los grupos de estudiantes de las tres áreas académicas mediante la prueba de ANOVA y post-hoc de Tukey. La prueba mostró diferencias significativas entre los grupos con respecto a las estrategias de creatividad ( $\mathrm{f}=8.811, \mathrm{n}=33, \mathrm{p}=.001$ ); en cambio, no mostró diferencias significativas en las estrategias globales de lectura $(f=0.0346, n=33, p=.966)$, ni en las estrategias de personalización ( $\mathrm{f}=2.983, \mathrm{n}=33, \mathrm{p}=.0658$ ) (figura 3 ). 

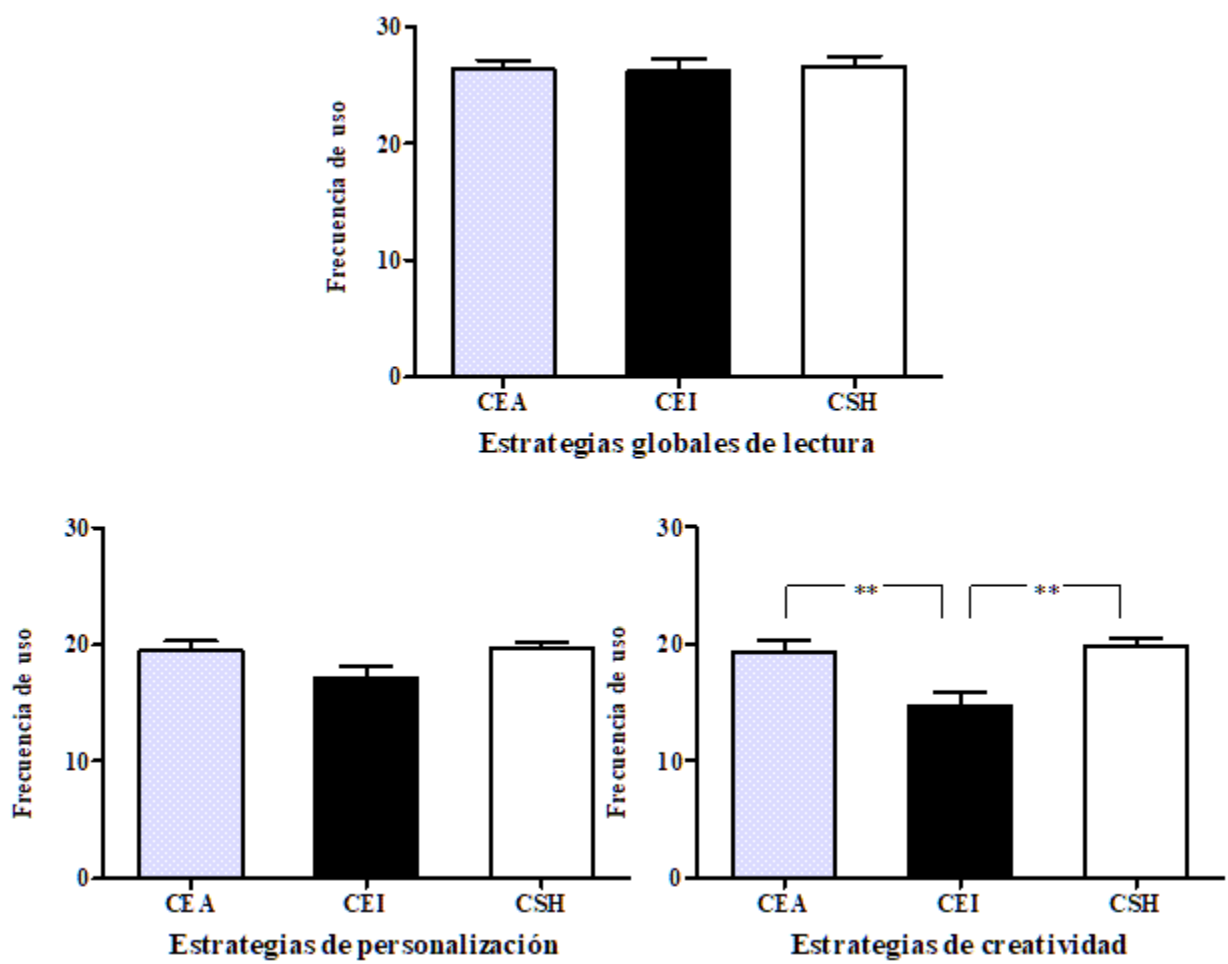

Figura 3. Comparación de uso de las estrategias lectoras de textos narrativos entre grupos de tres áreas académicas.

Nota: las barras muestran la media y las líneas la desviación estándar. $* * \mathrm{p}<.01$.

Fuente: elaboración propia.

Para los resultados de las estrategias lectoras de textos narrativos, comparamos la frecuencia de uso de las estrategias metacognitivas de comprensión de textos narrativos entre los grupos de estudiantes mujeres y hombres mediante la prueba de t de Student. La prueba no mostró diferencias significativas entre los grupos con respecto a las estrategias globales de lectura $(t=0.304, n=18, p=.764)$, ni en las estrategias de personalización $(t=0.619, n=18$, $p=.544)$, ni en las estrategias de creatividad $(t=0.756, n=18, p=.46)$ (figura 4). 

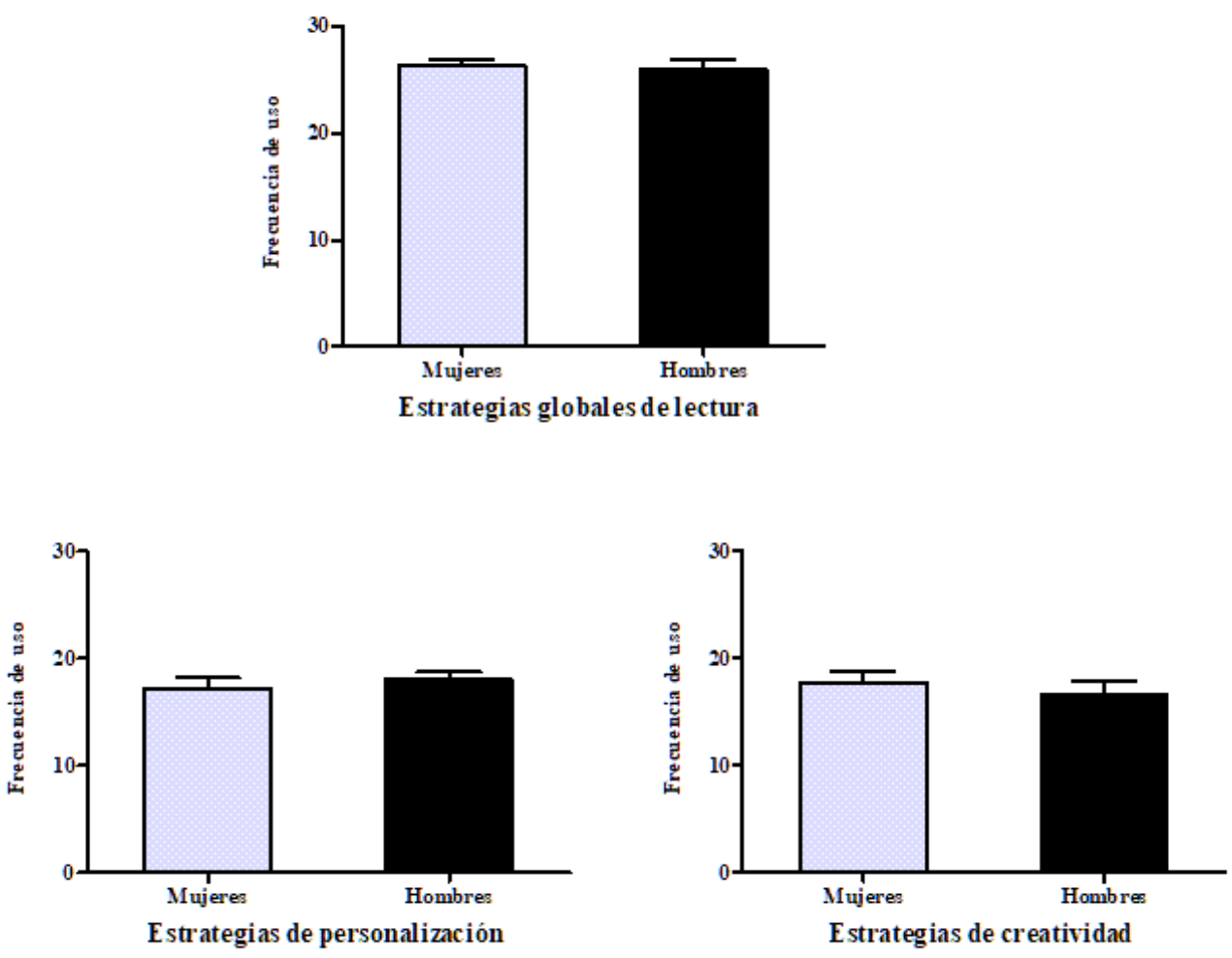

Figura 4. Comparación de uso de las estrategias lectoras de textos narrativos entre mujeres y hombres.

Nota: las barras muestran la media y las líneas la desviación estándar.

Fuente: elaboración propia.

En síntesis, de los dos experimentos sobre el comportamiento lector, los estudiantes pertenecientes al área de CEI (Cs. Exactas e Ingenierías) emplean menos las estrategias de creatividad en comparación con los estudiantes de las áreas de CEA (Cs. EconómicoAdministrativas) y CSH (Cs. Sociales y Humanidades) y esta diferencia es estadísticamente significativa. No existe diferencia estadísticamente significativa por género.

\section{Segunda fase}

Para los resultados de las respuestas abiertas a preguntas inferenciales de los estudiantes, comparamos el desempeño de los estudiantes por área académica y por tipo de inferencia. Para las preguntas de inferencias deductivas empleamos la prueba de ANOVA y post-hoc de Tukey. La prueba no mostró diferencias significativas entre los grupos con respecto a las inferencias deductivas ( $\mathrm{f}=0.1184, \mathrm{n}=33, \mathrm{p}=.889$ ). Para las preguntas de inferencias inductivas $\mathrm{y}$ abductivas empleamos la prueba de Kruskal-Wallis y post-hoc de Dunn. La prueba no mostró tampoco diferencias significativas entre los grupos con respecto a las inferencias inductivas ( $\mathrm{k}$ $=0.113, \mathrm{n}=33, \mathrm{p}=.9451)$, ni con las inferencias abductivas $(\mathrm{k}=1.457, \mathrm{n}=33, \mathrm{p}=.4825)$ (figura 5). 

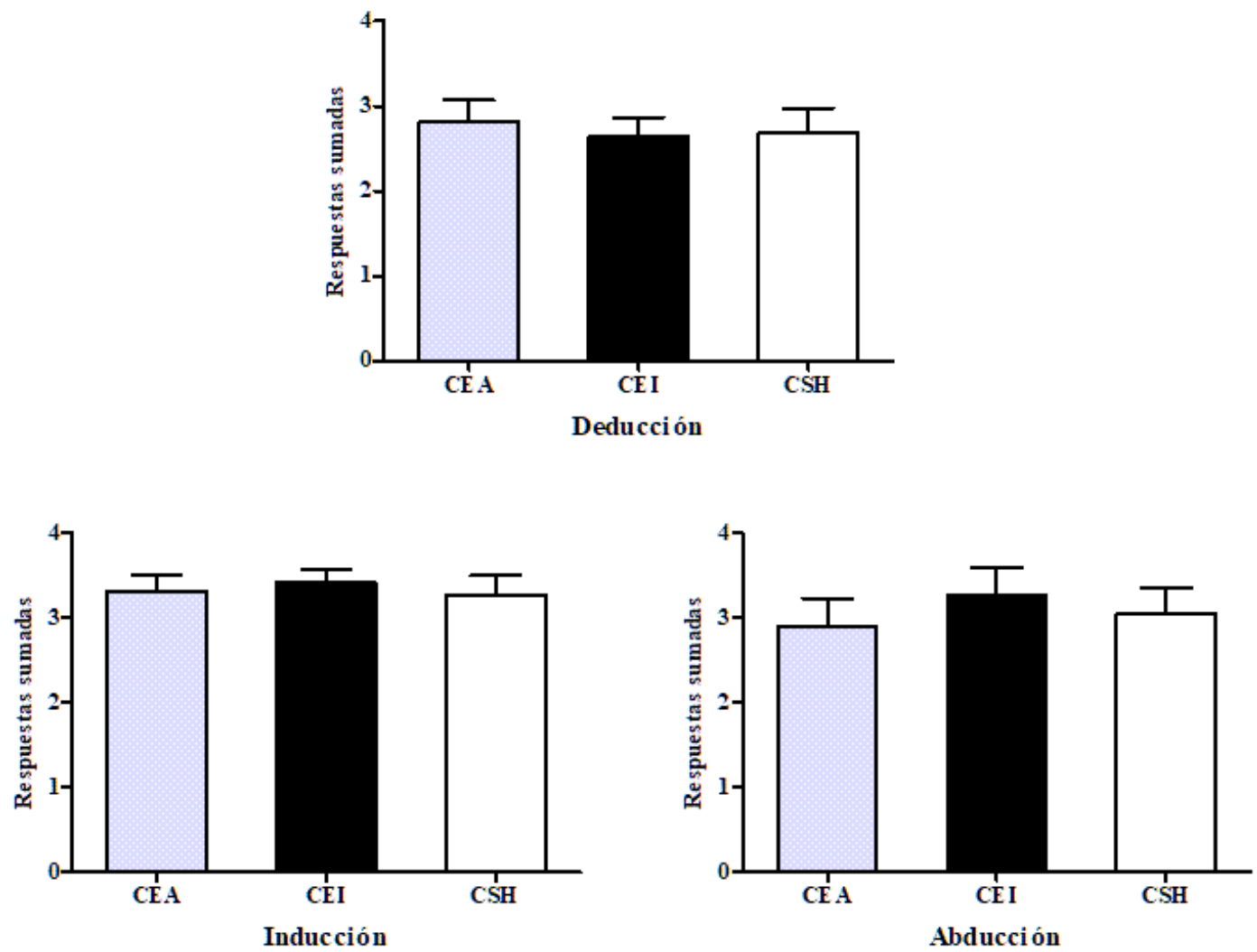

Figura 5. Comparación de las respuestas de los estudiantes por tipo de inferencia y por área académica.

Nota: las barras muestran la media y las líneas la desviación estándar.

Fuente: elaboración propia.

Al analizar las respuestas abiertas de los estudiantes encontramos algunos resultados adicionales, a saber: (1) el número de palabras empleadas por los estudiantes para argumentar sus respuestas tuvieron desde un mínimo de 10 palabras a un máximo de 23 palabras en promedio; (2) en la escritura a mano, notamos que varios estudiantes no pusieron los acentos en las palabras que ortográficamente deberían llevarlos.

Ahora, para los resultados de las respuestas de opción múltiple a preguntas inferenciales de los estudiantes, comparamos el desempeño de los estudiantes por género y por tipo de inferencia empleando la prueba $U$ de Mann-Whitney. No encontramos diferencias estadísticamente significativas con respecto a las inferencias deductivas $(U=37.5, n=18, p=$ .812 ), inductivas $(\mathrm{U}=39.0, \mathrm{n}=18, \mathrm{p}=.925)$, ni abductivas $(\mathrm{U}=30.5, \mathrm{n}=18, \mathrm{p}=.374)$ (figura $6)$. 

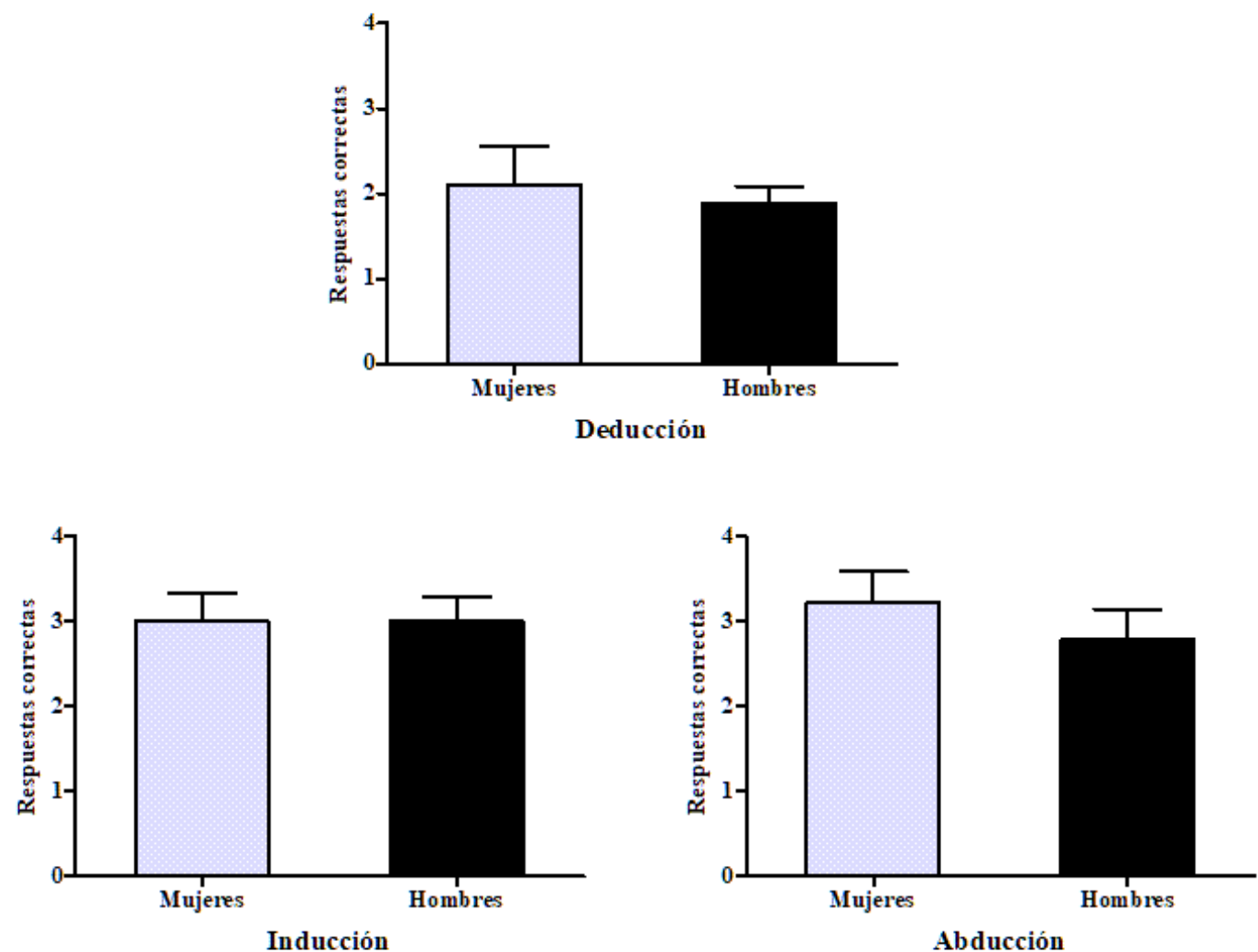

Figura 6. Comparación de las respuestas de los estudiantes por tipo de inferencia y por género. Nota: las barras muestran la media y las líneas la desviación estándar.

Fuente: elaboración propia.

La figura 5 permite observar que los estudiantes de la muestra clasificados por área académica obtuvieron niveles altos de desempeño en la prueba de comprensión lectora inferencial de respuesta abierta (eran 4 respuestas correctas posibles por tipo de inferencia). También la figura 6 permite observar un desempeño alto de los estudiantes con respuestas de opción múltiple en inferencias inductivas y abductivas, no así en inferencias deductivas en que tanto los hombres como las mujeres obtuvieron en promedio la mitad de respuestas correctas.

Discusión y conclusiones

En su mayoría, los resultados de los estudiantes en la prueba de comprensión lectora corresponden a niveles altos de desempeño tanto clasificados por área académica como por género (figuras 5 y 6); sin embargo, llama la atención que en esta investigación la mayoría de los estudiantes hayan obtenido la clasificación de "falsos lectores". Este hecho sugiere la necesidad de promover algún tipo de actividad en las clases con los estudiantes universitarios para que se lleve a cabo un análisis de las estrategias metacognitivas. 
Se puede mencionar que Larrañaga y Yubero (2015) explican que el CEMCoTeN pretende ser un instrumento para ayudar tanto a los estudiantes como a los docentes a detectar las estrategias metacognitivas de comprensión que no están activadas en la lectura de textos narrativos para poder planificar una intervención eficaz.

Incluso podría realizarse un estudio para conocer si la instrucción sobre el uso de las estrategias metacognitivas de estudiantes universitarios empleando textos narrativos influye en su desempeño; esto tomado como base la investigación de Espinosa (2020), quien afirma que la instrucción sobre el uso de estrategias de lectura de textos expositivos en estudiantes universitarios incide positivamente en su comprensión lectora.

Ahora, una explicación posible al hecho de que los estudiantes de CEI usen menos las estrategias de creatividad puede deberse no tanto a las acciones enunciadas en el instrumento (plantearse, analizar, imaginar, adivinar), que seguramente realizan de modo frecuente, sino a los objetos directos enunciados en los que recae la acción de tales verbos (los personajes, el relato). Tal vez si se les preguntara, cambiando el objeto directo con palabras como "los experimentos" o "las ecuaciones", por ejemplo, habrían respondido de otra forma.

Para los estudiantes considerados en esta investigación los conocimientos previos que tenían debido a su formación académica o sus preferencias de tipos de lectura debido a su género no parecen influir en su desempeño. Probablemente esto se debe a que es una actividad común para los universitarios inferir a partir de los contextos descritos en cada fragmento de texto (cf. Escudero y León, 2007). En trabajos futuros se podría ampliar la muestra para comparar estos resultados.

Al observar las tendencias en el desempeño en textos que solicitaban inferencias deductivas a partir del mismo fragmento de texto narrativo, parece que los estudiantes obtienen resultados más altos empleando respuestas abiertas (comparación por áreas) que de opción múltiple (comparación por género). Otro trabajo futuro podría indagar, específicamente en inferencias deductivas, la propensión a llegar a conclusiones inválidas cuando la persona conoce opciones de conclusiones inválidas (como los distractores de la prueba).

En este trabajo pudimos apreciar que, al emplear respuestas abiertas, los estudiantes pudieron expresar libremente su forma de comprender el texto y justificaron su conclusión en cada inferencia sugerida a partir de fragmentos de textos narrativos. De esta forma, coincidimos con Intraversato y Lucisano (2013) cuando explican que, aunque existen desventajas para los evaluadores debido al tiempo y al trabajo que implica la asignación de valor para las respuestas abiertas, son más las ventajas para el proceso de evaluación de la comprensión lectora, ya que 
los estudiantes expresan su proceso de razonamiento de forma escrita y las ideas que tienen sobre los textos (a diferencia de lo que sucede con las respuestas cerradas).

Uno de los resultados que no habíamos considerado como central en este estudio fueron los errores cometidos en la escritura a mano (falta de acentos en las palabras). Así, un trabajo futuro podría versar sobre lo siguiente: sabemos que la escritura de los estudiantes universitarios tiene una característica particular consecuencia de que los jóvenes suelen estar en contacto constante con los medios digitales de comunicación, a saber, un tipo de escritura disortográfica. GómezCamacho (2014) explica que el término escritura disortográfica se emplea "para la discrepancia intencionada con la ortografía académica que se produce entre hablantes competentes en la escritura mediada por ordenador a través de Internet y en los mensajes de texto a través de la telefonía móvil” (p. 24).

Asimismo, Gómez-Camacho y Gómez (2015) mencionan que uno de los rasgos frecuentes de la escritura disortográfica de los mensajes de texto en español de estudiantes universitarios es la omisión total o parcial de las tildes. Al analizar los textos producidos por los estudiantes universitarios en nuestro estudio, nos llamó la atención que sucediera el mismo fenómeno de la omisión de tildes en la escritura de mensajes de texto, en este caso, en la escritura de textos escritos a mano. Quizás otra posibilidad es que los hábitos de escritura disortográfica de los estudiantes tengan un efecto en sus hábitos de escritura y comprensión de textos argumentativos escritos a mano.

\section{Referencias}

Adler, J. E., y Rips, L. J. (Eds.) (2008). Reasoning. Studies of Human Inference and Its Foundations. Cambridge: Cambridge University Press.

Alderson, J. C. y Urquhart, A.H. (1988). This test is unfair: I'm not an economist. En P. L. Carrell, J. Devine, D. E. Eskey. (Eds.), Interactive approaches to second language reading (pp. 168-182). Cambridge: Cambridge University Press.

Arends, R. I. (2007). Aprender a enseñar. (7 ed.). México: McGraw Hill.

Brown, G., Bull, J. y Pendlebury, M. (1997). Assessing Student Learning in Higher Education. Londres: Routledge.

Butler, A. C. y Roediger, H. L. (2008). Feedback enhances the positive effects and reduces the negative effects of multiple-choice testing. Memory \& Cognition, 36(3), 604-616. https://doi.org/10.3758/MC.36.3.604 
Campos, J. M. (2016). Kazimierz Ajdukiewicz, las oraciones interrogativas y la racionalidad de los presupuestos al hacer una pregunta. Los modos del creer. Tópicos del Seminario, 36, 121-138.

Elche, M., Sánchez-García, S. y Yubero, S. (2019). Lectura, ocio y rendimiento académico en estudiantes universitarios del área socioeducativa. Educación XX1, 22(1), 215-237. https://doi.org/10.5944/educxx1.21548

Escudero, I. y León, J. A. (2007). Procesos inferenciales en la comprensión del discurso escrito: Influencia de la estructura del texto en los procesos de comprensión. Revista signos, 40(64), 311-336. https://dx.doi.org/10.4067/S0718-09342007000200003

Espinosa, A. (2020). Las estrategias de lectura y su incidencia en la comprensión lectora de estudiantes de una universidad pública del noroeste de México. RIDE Revista Iberoamericana para la Investigación y el Desarrollo Educativo, 11(21). https://doi.org/10.23913/ride.v11i21.689

Gilardoni, C. (2006). Valoración del libro y mecanismos de acercamiento a la lectura en los estudiantes universitarios. Serie Bibliotecología y Gestión de Información, 16, 1-46.

Gómez-Camacho, A. (2014). La norma disortográfica en la escritura digital. Didac, 63, 1925.

Gómez-Camacho, A. y Gómez, M. T. (2015). Escritura ortográfica y mensajes de texto en estudiantes universitarios. Perfiles Educativos, 37(150), 91-104.

Intraversato, A. y Lucisano, P. (2013). Gli anni di Eco. Riflessioni sull'uso di prove strutturate con risposte chiuse e aperte a margine di una ricerca sulla comprensione della lettura. ECPS Journal, 7, 23-43. https://doi.org/10.7358/ecps-2013-007-intr

Larrañaga, E. y Yubero S. (2015). Evaluación de las estrategias metacognitivas de comprensión de textos narrativos. Ocnos, $14, \quad$ 18-27. https://doi.org/10.18239/ocnos_2015.14.02

Méndez, J. M. (1995). Lógica de la relevancia. En Lógica: Enciclopedia Iberoamericana de Filosofía (pp. 237-270). Madrid: Trotta.

Méndez, S. (2006). Comprensión lectora y textos literarios: una propuesta psicopedagógica. Revista Educación, 30(1), 141-155.

Pearson, P. D. \& Cervetti, G. N. (2017). The Roots of Reading Comprehension Instruction. En S. E. Israel. (Ed), Handbook of Research on Reading Comprehension (2da ed., pp. 12-56). New York: The Guilford Press.

Pérez Zorrilla, M. J. (2005). Evaluación de la comprensión lectora: dificultades y limitaciones. Revista de Educación, num. Extraordinario, 121-138. 
Soler García, J. M. (2005). Autobiografía de José María Soler García. (Edición digital). Alicante: Biblioteca Virtual Miguel de Cervantes.

Steffensen, M. S., Joag-dev, C. y Anderson, R.C. (1979). A cross-cultural perspective on reading comprehension. Reading Research Quarterly, 15, 10-29.

Torres, M., Paz, K. y Salazar, F. G. (2006). Métodos de recolección de datos para una investigación. Boletín electrónico Ingeniería, 3. Universidad Rafael Landívar.

West, M. y Chew, H. (2015). La lectura en la era móvil: Un estudio sobre la lectura móvil en los países en desarrollo. París: UNESCO. 